\title{
Setae thickening in Daphnia magna alleviates the food stress caused by the filamentous cyanobacteria
}

\author{
Lukasz Wejnerowski • Slawek Cerbin • \\ Marcin Krzysztof Dziuba
}

Received: 11 October 2016/Accepted: 25 May 2017/Published online: 29 May 2017

(C) The Author(s) 2017. This article is an open access publication

\begin{abstract}
It is assumed that daphnids adjust the filter screen morphology in order to minimize the interference with cyanobacterial filaments. The aim of this study was to investigate the impact of filamentous cyanobacteria (Aphanizomenon gracile Lemmermann, Cylindrospermopsis raciborskii Woloszynska Seenaya et Subba Raju) on the thickness and length of setae of the third pair of thoracic limbs of Daphnia magna. The second objective was to assess whether the setae modifications could improve the performance of daphnids in the presence of cyanobacteria. Three clones of Daphnia magna Straus were cultured with: green algae; green algae with filaments of Cylindrospermopsis; and green algae with filaments of Aphanizomenon. The size and age of animals in the first reproduction cycle as well as the number of offspring were recorded. Setae thickness and length were measured in the central part of each endopodite. Additionally, we analyzed how the changes in setae morphology affect the fitness of experimental animals using the intrinsic rate of population increase calculated with the Euler-Lotka equation. The results showed that the thickness and length of setae increased
\end{abstract}

Handling Editor: Sabine Giessler.

L. Wejnerowski $(\varangle) \cdot$ S. Cerbin · M. K. Dziuba Department of Hydrobiology, Faculty of Biology, Adam Mickiewicz University, Umultowska 89, 61-614 Poznan, Poland

e-mail: wejner@amu.edu.pl in the presence of filamentous cyanobacteria. Moreover, cyanobacteria-induced setae thickening was positively correlated to the fitness of daphnids, which may indicate setae thickening as a phenotypic adaptation to cope with food stress caused by filamentous cyanobacteria.

Keywords Filtering apparatus - Life history · Mechanical interference $\cdot$ Morphology ·

Phenotypic plasticity

\section{Introduction}

Planktonic filter feeders such as Daphnia can be effective grazers of phytoplankton biomass in aquatic ecosystems. However, the efficiency of Daphnia grazing is considerably reduced when the phytoplankton is mainly composed of filamentous cyanobacteria, which clog the meshes of Daphnia filtration apparatus and reduce its food ingestion (Gliwicz and Siedlar 1980; Haney 1987; Lampert 1987). At high concentrations, filaments entangle inside daphnids' filtering chamber (Hartmann and Kunkel 1991) and thoracic appendages movements are considerably reduced. In such conditions, the food ingestion and clearance rate were extremely low (Sahuquillo et al. 2007), whereas the frequency of post-abdominal claw rejection movements was markedly increased (Burns 1968; Haney et al. 1995). Hence, the presence of filamentous 
cyanobacteria has a negative impact on the life of Daphnia. Experimental studies demonstrated that cyanobacterial filaments could slow down somatic growth of daphnids (Gliwicz 1990; Soares et al. 2009), as well as inhibit and reduce their reproduction (Repka 1997; Reinikainen et al. 1999; Kurmayer 2001). Other researchers revealed that Daphnia could abort eggs when filamentous cyanobacteria were present, and this phenomenon intensified at higher temperatures (Bednarska and Slusarczyk 2013). Bednarska with coworkers also found evidence that reduction of Daphnia fitness in response to cyanobacterial filaments strongly depends on filaments length (Bednarska et al. 2014). According to the aforementioned study, long filaments $(>50 \mu \mathrm{m})$ caused a greater reduction in somatic growth and fecundity in most clones of $D$. magna, in comparison with short filaments $(<50 \mu \mathrm{m})$.

However, daphnids exhibit phenotypic plasticity in their filter screen morphology, which reduces the negative effects of unfavorable food conditions. Several researchers revealed that in limited concentrations of food, Daphnia setae on the filtering appendages were longer (Koza and Kořinek 1985; Pop 1991), filter screen areas were enlarged (Lampert 1994), but mesh sizes were smaller (Brendelberger 1991). These modifications enhance the efficiency of food collection and were recognized as an adaptation to the low-food environment. The reduction in mesh sizes allows Daphnia to intercept small bacteria (Brendelberger 1991), which may compensate for unfavorable nutritional conditions. The presence of filamentous cyanobacteria caused decreased intersetal and intersetular distances in the filtering apparatus, and elongation of filtering setae in Daphnia (Macháček 1998; Bednarska and Dawidowicz 2007). The latter authors suggested that such morphological changes reduce the interferential effect of cyanobacterial filaments on the filtration process. When the meshes on filter screens are finer, food intake is based mainly on direct interception of particles rather than mechanical sieving, because viscous forces dominate the flow. In this case, filtering appendages function as paddles rather than sieves (Koehl 1995), and most of the seston particles flow tangentially to the surface of filter screens (Gerritsen et al. 1988).

On the other hand, smaller meshes and enlarged filter screens cause higher resistance during filtration and an increase in daphnids' energy requirements
(Lampert and Brendelberger 1996). The resistance may be even higher when the filaments of cyanobacteria interfere with the filtration process. In our recent study (Wejnerowski et al. 2015), we suggested that thicker filaments could interfere to a higher extent with filtration process in comparison with thinner ones. One can expect that in such a situation Daphnia should strengthen its filtering appendages to cope with higher resistance.

Although modifications of the filtration apparatus of Daphnia have been broadly studied on numerous Daphnia species (Macháček 1998; Repka et al. 1999a, b; Ghadouani and Pinel-Alloul 2002; Bednarska and Dawidowicz 2007), little has been known about the changes in the thickness of setae induced by filamentous cyanobacteria. Because the distance between setae decreases in response to the presence of cyanobacteria (Bednarska and Dawidowicz 2007) and the number of setae on filtering appendages is constant in adult animals (Pop 1991; Lampert and Brendelberger 1996), it is reasonable to expect the setae to become thicker in such conditions. In our opinion, thickening of setae, induced by the presence of filamentous cyanobacteria, makes filtering comb stronger, which enables it to effectively fulfill its function, especially if it lengthens under low-food conditions. We hypothesize that daphnids, in the presence of filamentous cyanobacteria, would possess thicker and longer setae than individuals grown without them. Moreover, if increasing setae strength (by their thickening) reduces the negative effects of cyanobacterial filaments, the response should be intensified when wider (more grazing-resistant) cyanobacteria are present. Therefore, this study aimed to verify: (1) whether Daphnia setae thicken and lengthen in the presence of filamentous cyanobacteria, (2) if induced by filamentous cyanobacteria change of setae occurs, does it relate to the better performance of Daphnia, and (3) whether morphological modifications of filtering appendages are more pronounced in the presence of cyanobacteria having thicker, more grazing-resistant filaments.

To test our hypotheses, we examined the response in overall fitness and filtering apparatus morphology of Daphnia magna to the presence of Aphanizomenon gracile or Cylindrospermopsis raciborskii, filamentous cyanobacteria that differ in thickness and stiffness and hence in resistance to grazing as well. 


\section{Methods}

Experimental organisms and culture conditions

Three clones of D. magna were sampled from a longterm laboratory culture (Bdem2), a fishless pond in the city park in Poznań (Wilson) and a fishless pond in the meadows near the city (Zoot). These clones were cultured in 1-L glass beakers that were filled with filtered $(0.45-\mu \mathrm{m}$, Whatman GF/C) and conditioned lake water (Lake Kierskie Duże, western Poland). The animals were cultured under defined food conditions (mixture of $0.5 \mathrm{mg} \mathrm{C} \mathrm{L} \mathrm{L}^{-1}$ Acutodesmus obliquus (Turpin) Hegewald et Hanagata (formerly called Scenedesmus obliquus Turpin Kützing) and $0.5 \mathrm{mg} \mathrm{C} \mathrm{L}^{-1}$ of Chlorella vulgaris Beyerinck, refreshed every second day), temperature $\left(20^{\circ} \mathrm{C}\right.$ ) and light (PAR: $44 \mu \mathrm{mol} \mathrm{m}{ }^{-2} \mathrm{~s}^{-1}$, 16:8 h L:D cycle) for several generations.

In this study, two species of filamentous cyanobacteria, A. gracile strain SAG 31.79 and C. raciborskii strain SAG 1.97, were used. According to Komárek (2013), the width of $A$. gracile ranges between 2.6 and $3.1 \mu \mathrm{m}$ and of $C$. raciborskii between 2.0 and $2.4 \mu \mathrm{m}$. Moreover, filaments of $A$. gracile are usually stiff, whereas those of $C$. raciborskii are often pliant and rarely twisted (Shafik et al. 2003). Filaments of both strains used in this study were straight without tendency to twist but very long filaments were slightly bent. The morphology of the strains used in our research was determined before the experiment. The filaments were similar in length, but they differed in thickness (Table 1). Moreover, this difference was statistically significant (Student's $t$ test: $t=11.7$, $d f=12, p<0.0001)$. This statistic was calculated based on 7 replicates for each species of cyanobacteria (each replicate represents mean of 30 randomly selected specimens). An earlier study revealed that these strains produce no cylindrospermopsin, and the exudates of both strains had no negative effect on daphnids (Wejnerowski et al. 2015). Moreover, our recent chromatographic tests (HPLC-DAD) showed that these strains do not produce microcystins LR, RR and YR (Wejnerowski et al. under review).

Cyanobacteria and green algae were cultured in the chemostats in WC medium (Guillard and Lorenzen 1972) pumped by a peristaltic pump (Ismatec, BVP Standard, Switzerland) in a phytotron (Conviron). Biomass of filaments and algal cells of each strain were harvested and subsequently separated from the
Table 1 Filament biomass of A. gracile (APH) and C. raciborskii (CYL) used in the experiment, and the results of the morphological characterization of cyanobacteria made before the experiment

\begin{tabular}{lll}
\hline Parameter & \multicolumn{2}{l}{ Treatment } \\
\cline { 2 - 3 } & $\mathrm{APH}$ & $\mathrm{CYL}$ \\
\hline Filament biomass $\left(\mathrm{mg} \mathrm{C}^{-1}\right)$ & \\
Mean $\pm \mathrm{SD}$ & $1.41 \pm 0.34$ & $1.27 \pm 0.12$ \\
Filament thickness $(\mu \mathrm{m})$ & \\
Mean $\pm \mathrm{SD}$ & $2.15 \pm 0.14$ & $1.56 \pm 0.03$ \\
Filament length $(\mu \mathrm{m})$ & \\
Mean \pm SD & $250.3 \pm 19.5$ & $271.5 \pm 36.3$ \\
Variability of filament & thickness \\
Min-max & $1.56-3.92$ & \\
Median & 1.98 & $1.18-2.01$ \\
Lower quartile & 1.88 & 1.54 \\
Higher quartile & 2.10 & 1.47 \\
Variability of filament & length & 1.64 \\
Min-Max & $35.2-757.5$ & \\
Median & 216.2 & $47.0-1235.5$ \\
Lower quartile & 145.1 & 236.4 \\
Higher quartile & 327.6 & 149.7 \\
\hline Concerning filament & 355.2 \\
\hline
\end{tabular}

Concerning filament biomass analysis, three replicates were distinguished for APH and CYL treatment. We used seven replicates for $\mathrm{APH}$ and $\mathrm{CYL}$ to characterize the strains of cyanobacteria morphologically

Filament thickness was measured on 30 randomly selected specimens in each replicate $(30 \times 7=210$ measurements $)$. Filament length was measured on 100 randomly selected specimens in each replicate $(100 \times 7=700$ measurements $)$

$S D$ means standard deviation

liquid media by centrifugation for $30 \mathrm{~min}$ at $3000 \mathrm{rpm}$ (Rotina 380R centrifuge, Germany). Collected pellets were re-suspended in filtered lake water before being used in the experiment.

\section{Design of the experiment}

The neonates of each clone, born on the same day and originated from the third generation of single Daphnia individuals, were used in the experiment. This procedure was followed in order to minimize the involvement of maternal effects on the experiment. Single daphnids were kept in glass tubes filled with $100 \mathrm{~mL}$ of filtered lake water. The light and temperature conditions were the same as for the algal cultures. To 
test the effect of filamentous cyanobacteria on the life history response and morphology of filtering appendages of Daphnia, a $3 \times 3$ factorial experiment was conducted. The animals from 3 clones were fed (food treatment) with a 1:1 green algae mixture of $A$. obliquus and Ch. vulgaris (controls, $1 \mathrm{mg} \mathrm{C} \mathrm{L}^{-1}$ in total), or 1:1 mixture of $A$. gracile filaments with the green algae (APH treatment, $2 \mathrm{mg} \mathrm{C} \mathrm{L}^{-1}$ in total), or 1:1 mixture of $C$. raciborskii filaments with the green algae (CYL treatment, $2 \mathrm{mg} \mathrm{C} \mathrm{L}{ }^{-1}$ in total). Each treatment contained seven replications, which in total gave 63 experimental units $(3$ food treatments $\times 3$ Daphnia clones $\times 7$ replicates). Daphnids were transferred every second day into clean tubes containing fresh food suspensions. In all treatments, the concentration of algal food was constant and far above the incipient limiting level $\left(1 \mathrm{mg} \mathrm{C} \mathrm{L}{ }^{-1}\right)$. The average values of cyanobacterial filament biomass for both treatments are summarized in Table 1. The carbon concentrations of algal cell suspensions or cyanobacterial filaments were estimated from readings of photometric light extinction at $750 \mathrm{~nm}$ (Metertech, SP-830 Plus, Taipei, Taiwan) using previously established carbon-extinction regressions. The determination of carbon was made with a Vario Max elemental analyzer (Elementar Analysensysteme $\mathrm{GmbH}$, Germany). The experiment was carried out until the birth of the first neonates. After the reproduction, the body length (SFR) of daphnids and the age at first reproduction were measured. The SFR was defined as the distance between the most posterior point on the head and the base of the junction of the tail spine and the carapace. The age of Daphnia females at first reproduction was determined with a 24-h accuracy. In addition, living neonates were counted. Observations of the daphnids and body length measurements were performed using the stereomicroscope Leica EZ4 (Germany) with an ocular lens micrometer.

As a measurement of Daphnia fitness, the intrinsic rate of population increase $(r)$ was used and calculated with the Euler-Lotka equation (Stearns 1992).

Preparation and measurements on filtering appendages

After the first reproduction cycle, each daphnid was fixed in $70 \%$ ethanol. The third pair of filter appendages (also referred to as phyllopods or flattened limbs) from each individual was dissected, immersed in a glycerin drop on a microscope slide, and covered with a cover glass. In total, we dissected and analyzed 126 filter appendages. The thickness and length of 15 filtering setae in the central region of each filtering limb was measured at $1000 \times$ or $100 \times$ magnification (respectively) using a light microscope Axioscope 2 Mot Plus (Carl Zeiss Light Microscopy, Germany) with a digital camera JENOPTIK ProgRes Speed Xt core 3 and ProgRes Image Capture Software (Jenoptik, Germany). The thickness of filtering setae was measured at two points of setae: the base (ST1) and the distal part (ST2) (Fig. 1). The setae length (SL) was measured according to the scheme in Fig. 1. A total number of measurements performed was 5670: 15 measurements $\times 2$ filtering limbs (from a single animal) $\times 3$ parameters $(\mathrm{SL}, \mathrm{ST} 1, \mathrm{ST} 2) \times 3$ treatments (control, APH, CYL) $\times 7$ replicates $\times 3$ Daphnia clones (Bdem2, Wilson, Zoot). In order to analyze the morphology of setae, we averaged all measurements of a given variable made on the single replicate of Daphnia. As a result, we obtained seven mean results for a single variable in each treatment. To eliminate the variations in setae morphology resulting from body size, the relative filtering setae length (RSL) was calculated using the method described by Pop (1991), in which the setae length was expressed as a percentage of the body length. The relative setae thicknesses (RST1 and RST2) were expressed here as

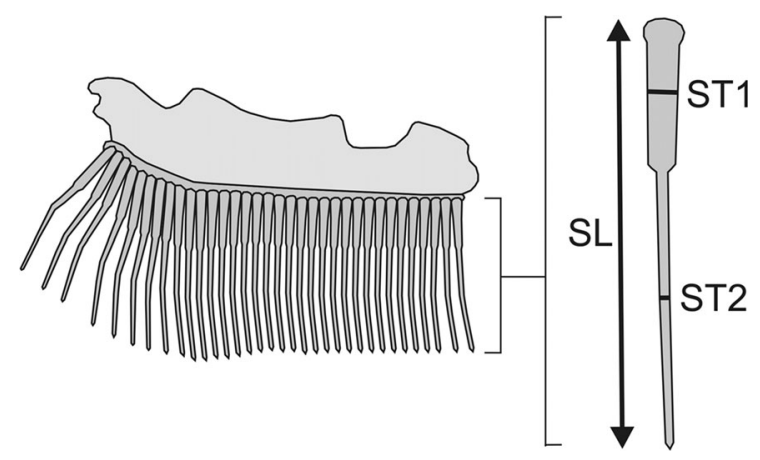

Fig. 1 Schematic picture of a single filtering appendage of Daphnia (left schema), and single filtering setae (right schema) with marked (black bold lines) measurement points of setae thickness at the base (ST1) and the distal part of setae (ST2) and setae length (SL). A double-headed arrow indicates how setae length was measured. Setae thickness and their length were measured on 15 filtering setae in the central part of each flattened limb 
setae thickness at a given measurement point to setae length ratio and multiplied by 1000 .

\section{Statistical analyses}

A two-way ANOVA was applied to analyze significant effects of experimental factors (food type, clone) on the size of animals at first reproduction (SFR), the absolute (ST1, ST2) and relative (RST1) setae thickness. Multiple pairwise comparisons of significant ANOVAs were made with post hoc Tukey's HSD test at the 0.05 significance level. In RST2 and RSL, the variances were not homogenous (Levene's test, $p<0.05$ ), and transformation did not improve the data distribution. Therefore, we decided to use the Generalized Least Squares (GLS) technique, which allows to control for unequal variances. The analysis of orthogonal contrasts was performed in order to compare the control versus cyanobacteria (contrast Cyano 1, regardless of the species of cyanobacterium) and CYL versus APH (contrast Cyano 2). The clones were divided into two orthogonal contrasts as well. First contrast consisted of the clone Bdem2 versus clones Wilson and Zoot (contrast Clone 1) and the second one of clone Wilson versus Zoot (contrast Clone 2). We used ANCOVA to search for the differences in fitness related to the filtering appendages morphology. All statistics and graphs were performed with the R statistical software version 3.0.2 ( $R$ Core Team 2013), which is under general public license.

\section{Results}

The size at first reproduction (SFR) was influenced by food type and differed among clones (food type $\times$ clone: $F_{(4,54)}=9.45, p<0.001$, Fig. $\left.2 \mathrm{a}\right)$. The SFR of Bdem 2 was reduced only in the A. gracile treatment (APH), while the Wilson clone exhibited reduced SFR in the presence of both species of cyanobacteria. Moreover, A. gracile caused even greater decrease in the SFR of Wilson clone in comparison with $C$. raciborskii (CYL). Daphnids of the Zoot clone grew smaller with cyanobacteria as well, but there were no differences between $A$. gracile or $C$. raciborskii treatments. Regardless of the clone, the size at first reproduction was under the strong influence of food type (ANOVA, main effect of food: $F_{(2,54)}=138.28$, $p<0.001$, Fig. 2b). The presence of either C. raciborskii or A. gracile led to a decrease in body size at first reproduction in comparison with control. In addition, daphnids grown with thicker A. gracile, were smaller at first reproduction than those cultured with $C$. raciborskii. The clone was also a significant source of variation in the SFR (main effect of clone: $F_{(2,54)}=10.93, p<0.001$, Fig. 2c). The animals of Bdem 2 and Wilson clones were smaller in comparison with those of Zoot, regardless of the food type.

The absolute values of the setae thickness at the base (ST1) indicated a different effect of the treatment on each clone (ANOVA, food type $\times$ clone: $F_{(4,54)}=13.97, \quad p<0.001$, Fig. 3a). The Bdem2 clone in the presence of $C$. raciborskii had thicker setae than in control and under APH treatment. Moreover, daphnids of the Wilson clone possessed either thinner or similar setae in comparison with control. There were no significant differences among treatments in the Zoot clone. Very similar results were found for the ST2 (Fig. 3b). The interaction between treatment and clone was significant (food type $\times$ clone, $\left.F_{(4,54)}=14.23, p<0.001\right)$, and the trends in treatments for each clone were parallel to ST1. Apparently, setae thickness at both measured places depended on the size of daphnids, and the increase in SFR was found to be associated with a significant increase in the ST1 (data log transformed, $R^{2}=0.23$, $b=0.321, t_{(61)}=4.259, p<0.001$, Fig. 4a) and increase in ST2 $\left(R^{2}=0.55, b=0.638, t_{(61)}=\right.$ 8.647, $p<0.001$, Fig. 4b). Using the relative setae thicknesses, we removed the correlation with the animal size and the pattern of change became consistent for all clones. With respect to the relative thickness of setae at base (RST1), the ANOVA revealed no significant interaction of food type and clone (food type $\times$ clone: $F_{(4,54)}=0.615, p=0.65$ ) (Table 2). However, the RST1 increased when cyanobacterial filaments were present in food in comparison with the control only with green algae (main effects of food type, $F_{(2,54)}=41.78, p<0.001$ ) (Fig. 5a; Table 2). The differences between CYL and APH treatments were not significant (Fig. 5b). The relative setae thicknesses differed markedly among all three clones of D. magna as well, irrespective of the food type (main effects of clone, $F_{(2,54)}=42.27$, $p<0.001)$. The Bdem 2 clone had the greatest RST1 $(16.012 \pm 0.316 \mathrm{SE})$, and it was followed by Zoot $(14.451 \pm 0.286 \mathrm{SE})$ and Wilson (13.594 \pm 0.258 
Fig. 2 Size of daphnids of each clone after first reproduction (SFR) fed on green algae (control), Aphanizomenon (APH), and Cylindrospermopsis (CYL). Means and standard errors are shown. Letters inside the bars indicate homogenous groups revealed by Tukey's HSD for pairwise comparisons $(p<0.05)$
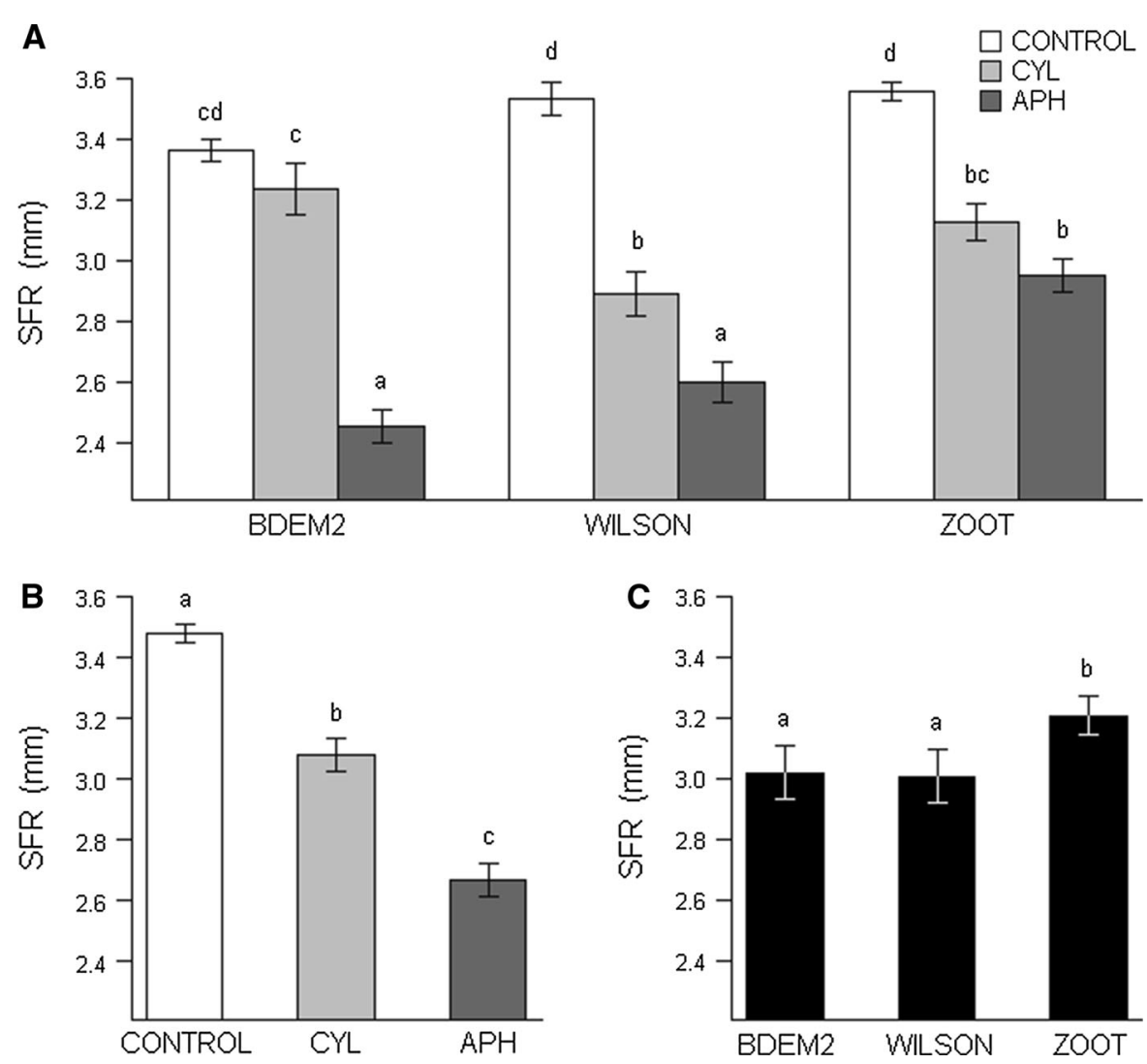

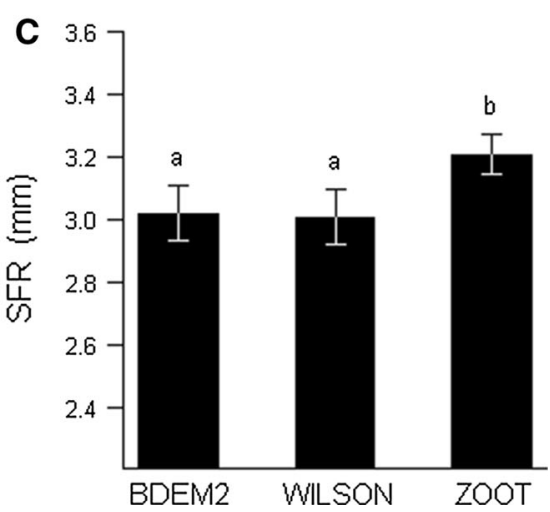

SE) (Table 2) clones. For the relative setae width at the distal part (RST2), we used the GLS to control for the unequal variances as described in methods section. The RST2 in controls were significantly lower than in both cyanobacteria treatments (Fig. 5c; Table 2), which is in opposition to the analyses of absolute setae widths. The differences between APH and CYL treatment were not significant (Fig. 5d). Considering the clones only, the RST2 of Bdem2 was similar to the ones of Wilson and Zoot clones; however, the Zoot clone exhibited greater relative setae thickness (RST2) than Wilson (Table 2).

The setae lengths (SL) were significantly different among food treatments (ANOVA, main effect food type: $\left.F_{(2,54)}=92.663, p<0.001\right)$. Daphnids in controls had longer setae than those in the cyanobacteria treatments (Fig. 6a). There were also significant differences between APH and CYL treatments (Fig. 6b; Table 2). The differences in absolute values of SL in food treatments depended on the clone (ANOVA, food type $\times$ clone, $F_{(4,54)}=5.11$, $p<0.01)$. The Bdem 2 clone had shorter setae in the presence of $A$. gracile than with $C$. raciborskii, while combined Wilson and Zoot clones showed no differences in response to both cyanobacteria species (Table 2).

Similarly to setae thicknesses, the length of setae was correlated with the SFR $\left(R^{2}=0.83, b=104.1\right.$, $t_{(61)}=16.8, p<0.001$, Fig. $\left.4 \mathrm{c}\right)$. In order to remove that effect, we used the relative setae length (RSL) in further analyses. However, we encountered problems with unequal variances like in relative setae thickness (RST2); thus, we used the GLS analyses. The data on setae length relative to the body length (RSL) revealed pattern opposite to absolute setae length (SL). The RSL increased in comparison with control (Fig. 6c; Table 2). Moreover, daphnids fed with A. gracile (APH) showed a significant increase in the RSL in comparison with CYL treatment with $C$. raciborskii (Fig. 6d). This is again in contradiction to the results of absolute SL. There were also differences among clones with the Bdem 2 clone being smaller than combined Zoot and Wilson clones, and at the same time the last two having similar RSL (Table 2). 


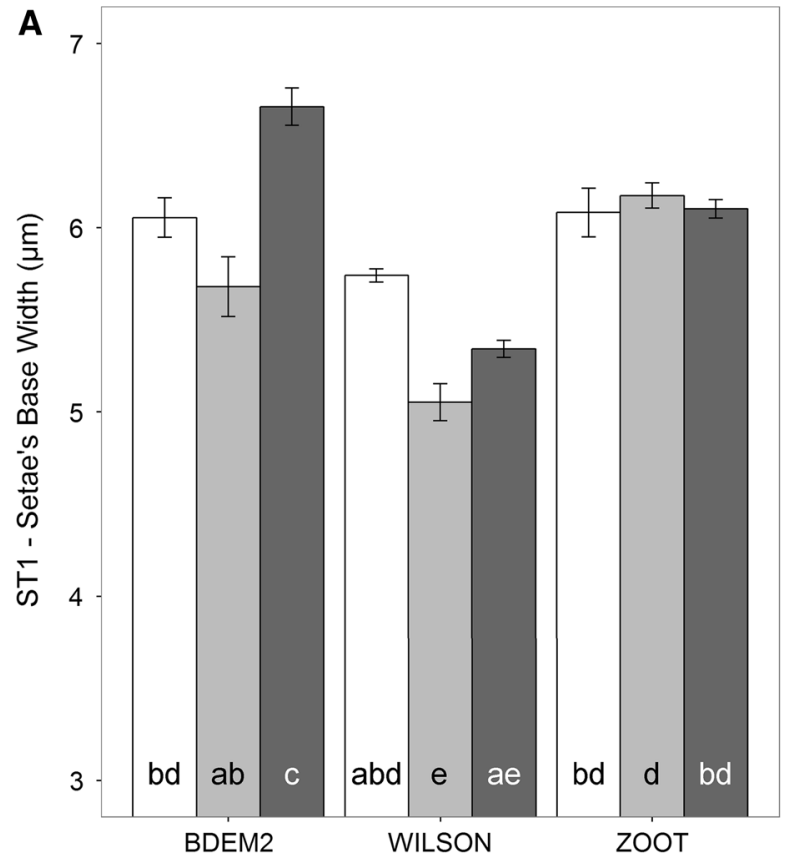

Fig. 3 Absolute setae thickness at A the base, ST1 and B distal part, ST2 of setae of D. magna of each clone (Bdem2, Wilson, and Zoot) fed on green algae (control), Aphanizomenon (APH),

The relation between intrinsic rate of increase and relative setae thicknesses (RST1 and RST2) differed between controls and cyanobacteria treatments (ANC OVA, significant interactions between covariates and treatment effects, respectively: $F_{(2,57)}=6.527$, $p<0.01 ; F_{(2,57)}=6.296, p<0.001$ ) (Fig. 7a, b). The assumptions for ANCOVA were not fulfilled; thus, we further analyzed the data using regressions. The RST1 in controls were not related with fitness (adjusted $R^{2}=-0.05, p=0.922$ ), while in APH and CYL treatments it was positive and significant (adjusted $R^{2}=0.368, p<0.01 ; R^{2}=0.556, p<$ 0.001 , respectively). The RST1 values were greater in CYL than in APH treatment (RST1: $t=4.965$, $p<0.001$ ), what means that daphnids grown with thinner $C$. raciborskii had greater intrinsic rate of increase than those grown with thicker A. gracile.

The relative thickness of the distal part of setae (RST2) was positively related with intrinsic rate of increase only in the CYL treatment (adjusted $R^{2}=0.46, p<0.001$ ), while the slope for APH treatment was not significant (adjusted $R^{2}=0.07$, $p<0.123$ ) similarly to the control. However, the

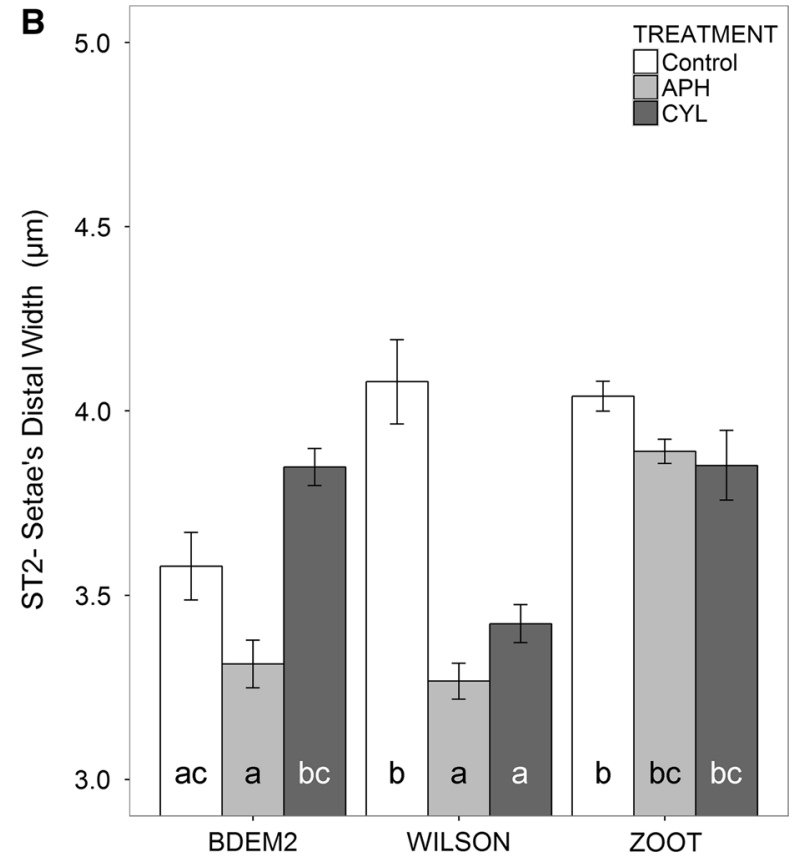

and Cylindrospermopsis (CYL). Means and standard errors are shown. Letters inside the bars indicate homogenous groups revealed by Tukey's HSD for pairwise comparisons $(p<0.05)$

RST2 were greater in CYL than in APH treatment (RST2: $t=4.633, p<0.001$ ), similarly to RST1.

\section{Discussion}

Morphological modifications of filtering apparatus in Daphnia exhibited in the presence of filamentous cyanobacteria are recognized as adaptive phenotypic plasticity allowing animals to minimize the interference of filaments with filtration process and to withstand food stress (Macháček 1998; Repka et al. 1999a, b; Ghadouani and Pinel-Alloul 2002; Bednarska and Dawidowicz 2007). In the present study, we expected that daphnids would possess thicker and longer setae in the presence of filamentous cyanobacteria. We assumed that induced thickening of setae would strengthen the filtering comb and improve its functioning. However, we observed that clones differed in morphological response to the presence of different species of cyanobacteria (Fig. 3). For example, the Bdem 2 clone had thicker setae in the presence of C. raciborskii than in A. gracile or control, the 

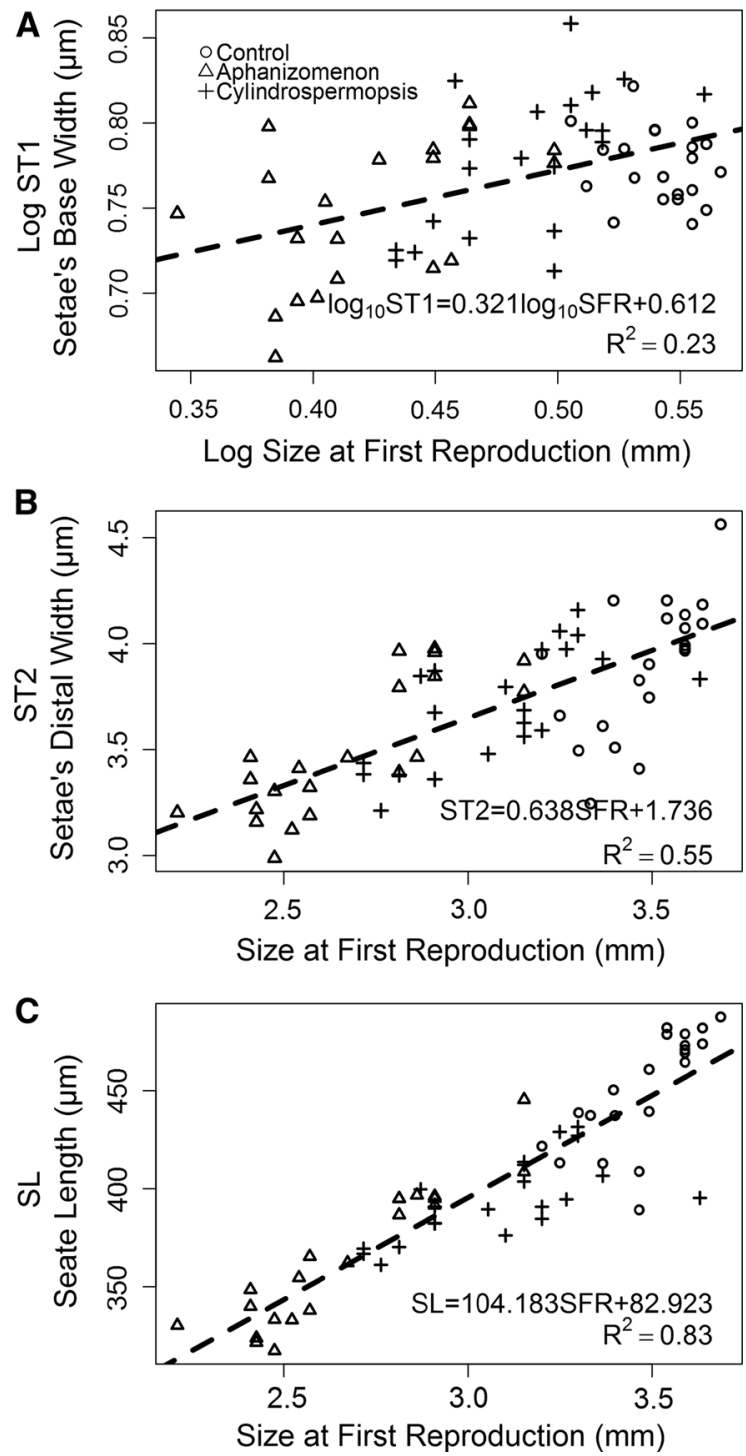

Fig. 4 Regression curves of pooled data from all treatments showing relations between size at first reproduction (SFR) and a $\log$ ST1—setae thickness at the base, b ST2 - setae thickness at the distal part, and c SL-setae length. Open circles correspond to control, triangles to Aphanizomenon (APH) treatment, and pluses sign to Cylindrospermopsis (CYL) treatment

Wilson clone had thinner setae than in control, and the Zoot clone did not exhibit any significant differences. These results may be explained by the size of daphnids, here measured at the day of first reproduction (SFR). Filamentous cyanobacteria have a negative effect on daphnids and reduce their size (Gliwicz 1990), which is correlated with all three morphological parameters of filtering appendages measured in our study (Fig. 4). However, different clones show variable resistance to cyanobacteria and that is why the results of absolute values reflect the variability in overall resistance to cyanobacteria and not the reaction to the mechanical factors only. Perhaps, clonal variation in response to food stress caused by cyanobacterial filaments was due to a different history of examined clones. Many previous studies have shown that Daphnia genotypes experienced with cyanobacteria in the past can have a greater performance at the next encounter with cyanobacteria in comparison with not experienced genotypes (Lemaire et al. 2012; Chislock et al. 2013). Although we cannot be sure whether our clones had contact with filamentous cyanobacteria before they have been acquired, the ponds where the Wilson and Zoot clones originate from never-experienced cyanobacterial blooms. Nevertheless, intraspecific variation observed in this study among clones of D. magna indicates a complexity in cyanobacteria-Daphnia interactions that are size related. Gliwicz and Lampert (1990) showed that the inhibitory effects of filaments are body size dependent. Here, in order to remove the correlation with daphnids size we used the relative measures of thicknesses and length of setae. The relative setae thickness of the third pair of thoracic limbs of $D$. magna increased in the presence of $C$. raciborskii and A. gracile in comparison with control. The increase was evident for setae bases (RST1) and at their distal parts (RST2) in all examined clones of D. magna (Fig. 5). Considering the changes of the relative setae thickness and length, we can see that under cyanobacterial stress they were not reduced proportionally to the body size reduction. This indicates the importance of maintaining the optimal shape of filtering apparatus even in adverse conditions where body size is reduced. However, the setae elongation depended on species of cyanobacteria as $A$. gracile induced an increase in setae length while the presence of $C$. raciborskii filaments was negligible (Fig. 6d).

The response of daphnids to the presence of A. gracile confirmed the results of previous field investigations, which indicated that the domination of filamentous cyanobacteria (Aphanizomenon sp.) in phytoplankton caused an increase in setae length of the third pair of thoracic limbs in Daphnia galeata Sars (Macháček 1998). This author interpreted the elongation of setae (relative to the body length) induced by the presence of filamentous cyanobacteria as daphnids' 
Table 2 Results of contrasts analyses of relative setae base thickness (RST1), relative setae distal thickness (RST2), setae length (SL), and relative setae length (RSL)

\begin{tabular}{|c|c|c|c|c|c|c|c|c|}
\hline \multirow[t]{2}{*}{ Contrast } & \multicolumn{2}{|l|}{ RST1 } & \multicolumn{2}{|l|}{ RST2 } & \multicolumn{2}{|l|}{ SL } & \multicolumn{2}{|l|}{ RSL } \\
\hline & $t$ & $p$ & $t$ & $p$ & $t$ & $p$ & $t$ & $p$ \\
\hline Cyano 1 & 9.12 & $* * *$ & 6.92 & $* * *$ & -12.86 & $* * *$ & 2.32 & $*$ \\
\hline Cyano 2 & -0.51 & ns & -1.33 & ns & 4.46 & $* * *$ & -5.32 & $* * *$ \\
\hline Clone 1 & -8.61 & $* * *$ & -1.67 & ns & 5.10 & $* * *$ & 2.36 & $*$ \\
\hline Clone 2 & 3.21 & $*$ & 2.01 & $*$ & 4.12 & $* * *$ & -0.22 & ns \\
\hline Cyano 1 versus Clone 1 & -0.83 & ns & -1.72 & ns & -2.16 & $*$ & -0.72 & ns \\
\hline Cyano 2 versus Clone 1 & -1.19 & ns & -1.72 & ns & -2.55 & $*$ & 2.78 & $* *$ \\
\hline Cyano 1 versus Clone 2 & 0.54 & ns & 2.34 & $*$ & 2.71 & $* *$ & -0.03 & ns \\
\hline Cyano 2 versus Clone 2 & -0.18 & ns & 0.10 & ns & -1.36 & $\mathrm{~ns}$ & -0.66 & ns \\
\hline
\end{tabular}

Contrasts abbreviations mean: Cyano 1-control versus CYL + APH, Cyano 2-CYL versus APH, Clone 1-Bdem2 versus Zoot + Wilson, Clone 2-Zoot versus Wilson

Asterisks indicate significant differences between compared groups

$* p<0.05$; ** $p<0.01$; *** $p<0.001)$

adaptive response to poor-quality food conditions. Setae elongation allows daphnids to enlarge the filter screen area and therefore enhances the filtering efficiency under unfavorable food conditions (Lampert 1994; Ghadouani and Pinel-Alloul 2002). However, morphological modifications aiming at increasing the filtering rate by increasing the appendages' surface may constitute a trap for daphnids when cyanobacterial filaments are present. The cyanobacterium C. raciborskii did not cause such a strong response in the setae elongation like $A$. gracile did. We offer two possible explanations for this result. Firstly, filaments of $A$. gracile have lower nutritional value than $C$. raciborskii or they produce toxins and thus have a more negative impact on the fitness of D. magna. We investigated our cyanobacteria strains for the presence of microcystins (see methods) and have not found any. Nevertheless, these toxins are not common in A. gracile or $C$. raciborskii and these species are potential producers of saxitoxin (Zanchett and Oliveira-Filho 2013; Cirés and Ballot 2016). Moreover, they can produce potentially toxic lipopolysaccharides, which are part of a cell wall (Stewart et al. 2006). However, our earlier study revealed that exudates of both strains used in this work did not negatively affect daphnids (Wejnerowski et al. 2015). Unfortunately, we do not have data concerning the differences in the nutritional status of both species. Secondly, A. gracile filaments could have been more harmful to daphnids because they were thicker than the filaments of $C$. raciborskii, while the biomass of both strains was on a similar level during the experiments (Table 1). Thickness-related resistance of filaments to grazing could be supported by the results of our previous study, in which we revealed that a share of thin filaments $(<2.5 \mu \mathrm{m})$ of the same strain of $A$. gracile decreased, while that of thick filaments $(>2.5 \mu \mathrm{m})$ increased during D. magna grazing (Wejnerowski et al. 2015). Moreover, filament thickness correlates positively with cell wall thickness (Wejnerowski et al. 2016), which determines physical properties of cyanobacterial filaments, especially their stiffness (Hoiczyk and Hansel 2000). According to DeMott (1995), the stiffness of food particles could determine the feeding efficiency of Daphnia, and stiffer or harder food particles could be grazed less effectively than the flexible ones. Hence, thicker filaments with thicker cell walls are probably stiffer and therefore more resistant to grazing than thinner ones.

Prior to the experiment, we assumed that thickening will strengthen the setae, a mechanism by which daphnids could minimize the negative effects due to mechanical grazing inhibition. Thicker and therefore stronger setae could effectively fulfill their role in food collection even when filaments interfered with and clog in Daphnia filtration apparatus. Since filaments need to be aligned along the food groove before ingestion (Hartmann and Kunkel 1991), strengthening 

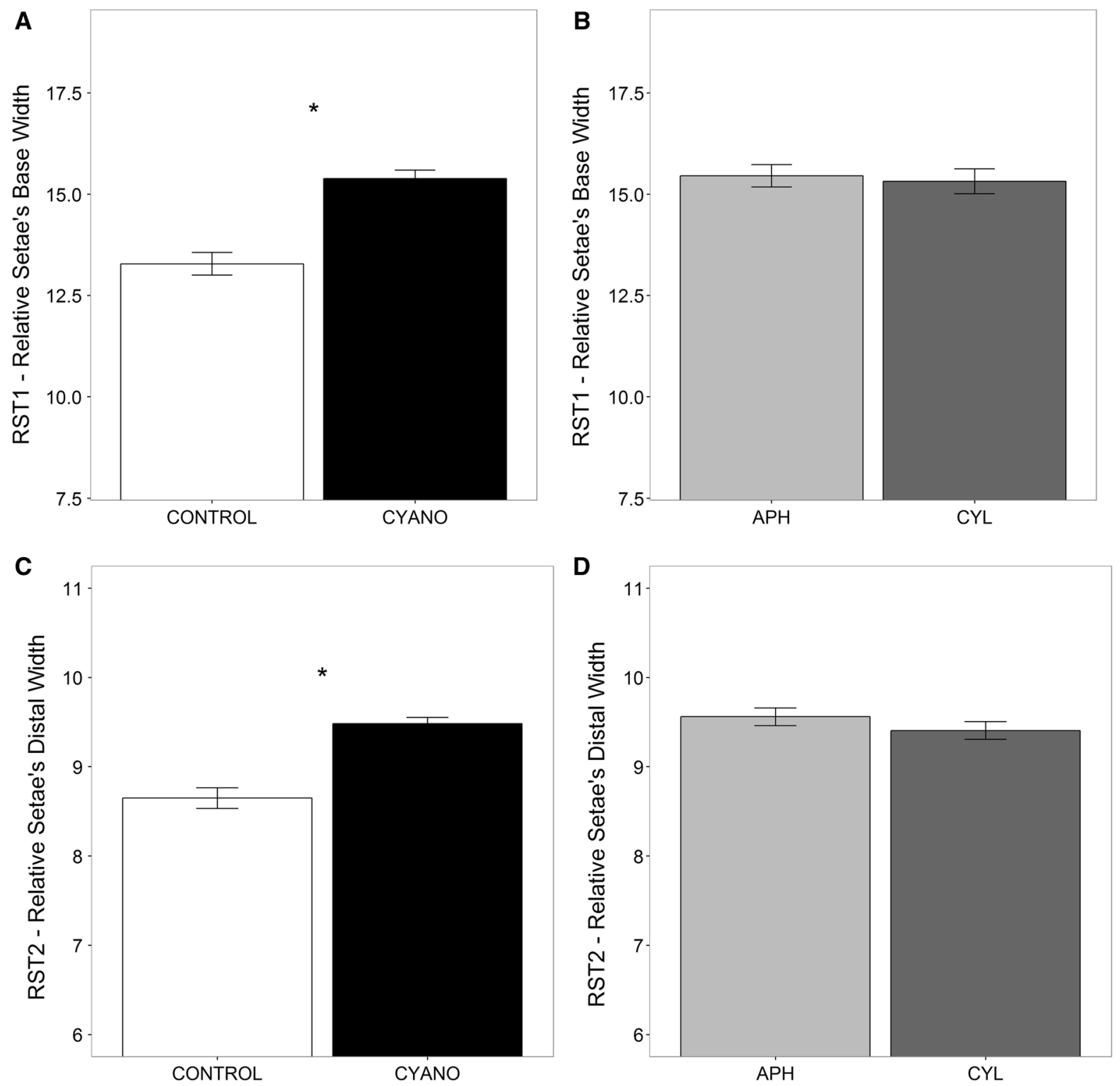

Fig. 5 Contrasts analyses of relative setae thickness at the base (RST1, upper panel) and a distal part (RST2, lower panel) of $D$. magna. Subplots a and c compare control (Daphnia fed on green algae and cyanobacteria treatments (contrast Cyano 1), and

of setae by their thickening could allow effective sweeping of grazing-resistant food particles like cyanobacterial filaments and clumping them together inside the filtering chamber. To support this assumption, future research should include measuring of clearance and ingestion rates in daphnids. The effective sweeping and clumping could improve ingestion of particles formed with filaments. In addition, we

subplots $\mathbf{b}$ and $\mathbf{d}$ show results of comparison between Aphanizomenon (APH) and Cylindrospermopsis (CYL) treatments (contrast Cyano 2). Means and standard errors are shown. An asterisk indicates significant differences $(p<0.05)$

predicted stronger thickening of setae in daphnids fed with A. gracile filaments. Both species of cyanobacteria-induced thickening of setae in daphnids; however, there were no significant differences between treatments with thick filaments of A. gracile and $C$. raciborskii with thinner filaments. The only significant response was observed in relative setae length in $A$. gracile treatment, where setae were relatively longer 

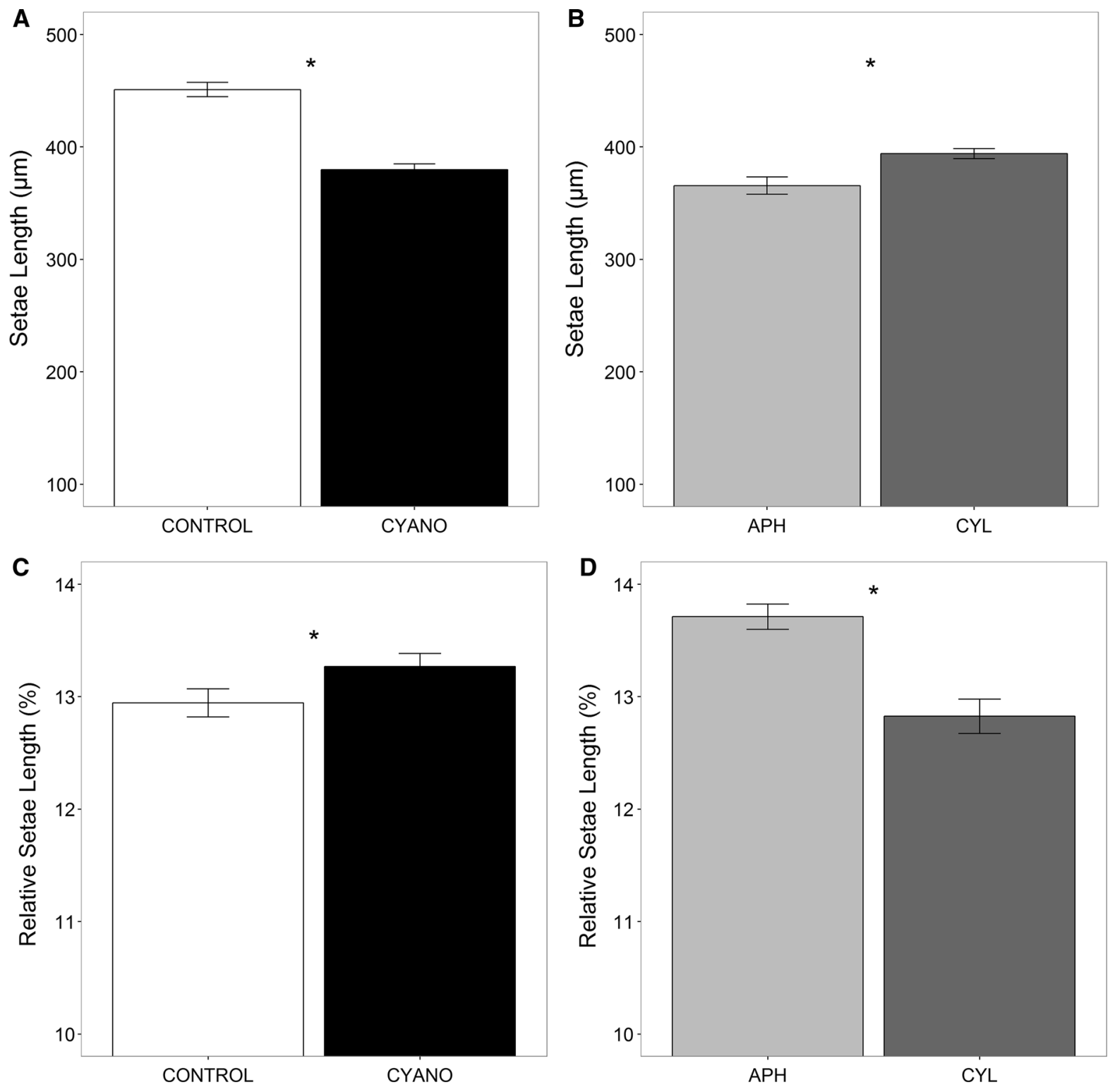

Fig. 6 Results of the analysis of contrasts for the absolute (SL, upper panel) and relative (RSL, lower panel) setae length. The groups were compared in terms of control versus cyanobacteria regardless of the species of the cyanobacterium (subplots a and

than in control or Cylindrospermopsis, what increased the appendages' relative surface.

Assuming our original explanation was true (setae thickening alleviates the negative effects of cyanobacterial filaments), we would be able to recognize positive responses of daphnids' life history and consider described changes in setae morphology as an adaptive feeding strategy to cope with

c, contrast Cyano 1), and between Aphanizomenon (APH) versus Cylindrospermopsis (CYL) treatments (subplots $\mathbf{b}$ and $\mathbf{d}$, contrast Cyano 2). Means and standard errors are shown. An asterisk indicates significant differences $(p<0.05)$

cyanobacterial filaments. In order to test this hypothesis, we calculated the intrinsic rate of increase and related it to the morphological characteristics of filtering appendages. It appeared that in controls the thickness of setae was not related to the intrinsic rate of increase. However, there was a significant positive relation between setae thickness and $r$ in both cyanobacterial treatments, which suggests that 
A

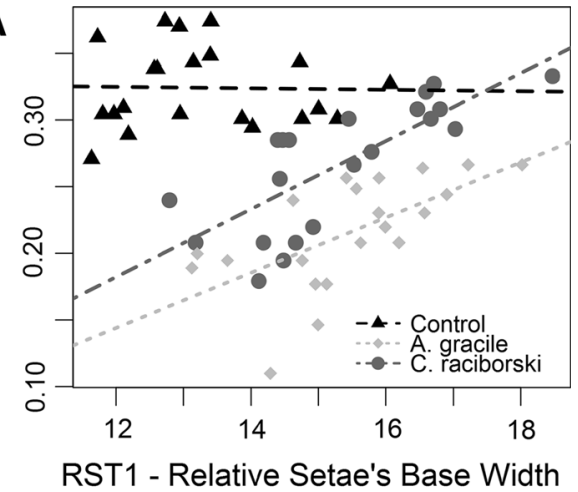

B

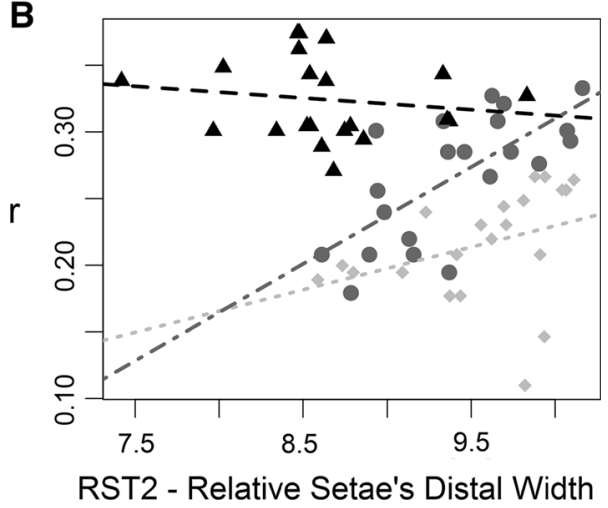

Fig. 7 Results of ANCOVA-relation between measured parameters, setae thickness at base expressed in setae length (RST1) or distal part of setae expressed in setae length (RST2) and intrinsic rate of increase $(r)$ (a, b, respectively). Black triangles and line correspond to control, light gray rhombuses and line to Aphanizomenon (APH) treatment and dark gray circles and line to Cylindrospermopsis (CYL) treatment

thickening setae might potentially increase the fitness when filamentous cyanobacteria are present. Our study presents only significant correlation between setae morphology and fitness but cannot be a proof of causation since there could be other factors influencing Daphnia performance. However, it is striking that the relation manifested only in the presence of filamentous cyanobacteria. It seems that the investment in stronger and larger filtering appendages is worthwhile when the overall effect of cyanobacteria is negative. Further studies are needed to test the effect of setae thickening on the fitness of daphnids simultaneously taking into account setae length as an independent factor. The design of experimental setup disentangling the morphology and the whole Daphnia performance, making setae morphology independent factor, might be quite problematic. One of possible solutions is to find Daphnia clones that do not alter the morphology of their setae in the presence of filamentous cyanobacteria and compare their performance with the clones that have a plastic response.

We sought to answer the key question: What triggered the observable morphological changes in filtering appendages. Undoubtedly, an increase in the setae length is a useful strategy to maximize the uptake of food particles when cyanobacteria are absent and the amount of food is limited (Lampert 1994). Daphnids, by elongating the setae, increase the filtering area and compensate for the lack of food. This adaptive mechanism is reflected in the increase in filtering rate (Stuchlik 1991; Lampert 1994). However, when cyanobacterial filaments occur in water (especially at a high biomass) the functioning of filtering appendages could be substantially impaired due to interference with filaments (Gliwicz and Siedlar 1980; Haney 1987). In their presence, the filtering efficiency substantially decreases (Zánkai and Ponyi 1986; Hawkins and Lampert 1989) and daphnids' filter mesh size of the filter screens is reduced what alleviates the mechanical interference (Bednarska and Dawidowicz 2007). However, when filtering appendages act like paddles, the forces acting on them are much larger and daphnids should strengthen their setae. We showed that increase in setae thickness is positively related to the fitness of Daphnia. However, this positive relation can be limited by higher biomass of filaments, which is recognized as one of the several main factors shaping filtration efficiency and, in consequence, the fitness of daphnids (Gliwicz 1990).

Setae thickening in the presence of cyanobacteria could also be crucial in regulating the intersetal distances in filtering appendages of Daphnia. The distance between setae bases decreased when cyanobacterial filaments were present, and this change together with the reduction of the filter mesh size (intersetular distances) may enable daphnids to minimize the interference of filaments with filtering apparatus (Bednarska and Dawidowicz 2007). Based on our findings on the thickening of setae in the presence of cyanobacteria and on the number of setae being constant in adult daphnids (Pop 1991), we are inclined to attribute the decrease in intersetal distances, observed by Bednarska and Dawidowicz (Bednarska and Dawidowicz 2007), to setae thickening. The confirmation of it might also be obtained by 
testing the relationship between setae thickness and the distance between setae.

\section{Conclusions}

To conclude, an observable increase in setae thickness of the third pair of thoracic limbs in the presence of filamentous cyanobacterial indicates a (hitherto not described) morphological change in daphnids in response to food stress. When the effect of body size was removed, all clones invested into thicker setae in the presence of cyanobacterial filaments. We also found a positive correlation between setae thickening and intrinsic rate of natural increase in daphnids, what may suggest that this type of a morphological response increases the fitness of daphnids when the overall effect of cyanobacteria is negative. It is necessary to continue this study using a range of concentrations of cyanobacterial filaments in order to support or disprove the adaptive character of setae modifications in response to the presence of cyanobacteria. In addition, we encourage other researchers to test whether other species of the genus Daphnia, especially the lakeside ones, also thicken their setae of the third pair of thoracic limbs in the presence of cyanobacterial filaments.

Acknowledgements The financial support for this work was provided by the National Science Centre in Poland by the Grant No. UMO-2012/07/N/NZ8/00166. The authors would like to thank Anna Kozłowska for laboratory assistance, Lechosław Kuczyński for statistical advice, and Michał Woszczyk for the analyzes of the carbon content in algal and cyanobacterial cultures, Piotr Maszczyk for comments and suggestions about the earlier version of the manuscript. LW is a beneficiary of National Science Centre in Poland scholarship for Ph.D. Students No. UMO-2016/20/T/NZ8/00303.

Open Access This article is distributed under the terms of the Creative Commons Attribution 4.0 International License (http:// creativecommons.org/licenses/by/4.0/), which permits unrestricted use, distribution, and reproduction in any medium, provided you give appropriate credit to the original author(s) and the source, provide a link to the Creative Commons license, and indicate if changes were made.

\section{References}

Bednarska A, Dawidowicz P (2007) Change in filter-screen morphology and depth selection: uncoupled responses of
Daphnia to the presence of filamentous cyanobacteria. Limnol Oceanogr 52:2358-2363. doi:10.4319/1o.2007.52. 6.2358

Bednarska A, Slusarczyk M (2013) Effect of non-toxic, filamentous cyanobacteria on egg abortion in Daphnia under various thermal conditions. Hydrobiologia 715:151-157. doi:10.1007/s10750-012-1424-2

Bednarska A, Pietrzak B, Pijanowska J (2014) Effect of poor manageability and low nutritional value of cyanobacteria on Daphnia magna life history performance. J Plankton Res 36:838-847. doi:10.1093/plankt/fbu009

Brendelberger H (1991) Filter mesh size of cladocerans predicts retention efficiency for bacteria. Limnol Oceanogr 36:884-894. doi:10.4319/lo.1991.36.5.0884

Burns CW (1968) Direct observations of mechanisms regulating feeding behavior of Daphnia, in lakewater. Int Rev Gesamten Hydrobiol Hydrogr 53:83-100. doi:10.1002/ iroh.19680530104

Chislock MF, Sarnelle O, Jernigan LM, Wilson AE (2013) Do high concentrations of microcystin prevent Daphnia control of phytoplankton? W Res 47:1961-1970. doi:10.1016/ j.watres.2012.12.038

Cirés S, Ballot A (2016) A review of the phylogeny, ecology and toxin production of bloom-forming Aphanizomenon spp. and related species within the Nostocales (cyanobacteria). Harmful Algae 54:21-43. doi:10.1016/j.hal.2015.09.007

DeMott WR (1995) The influence of prey hardness on Daphnia's selectivity for large prey. Hydrobiologia 307: 127-138. doi:10.1007/BF00032004

Gerritsen J, Porter KG, Strickler JR (1988) Not by sieving alone: observations of suspension feeding in Daphnia. Bull Mar Sci 43:366-376

Ghadouani A, Pinel-Alloul B (2002) Phenotypic plasticity in Daphnia pulicaria as an adaptation to high biomass of colonial and filamentous cyanobacteria: experimental evidence. J Plankton Res 24:1047-1056. doi:10.1093/ plankt/24.10.1047

Gliwicz ZM (1990) Daphnia growth at different concentration of blue-green filaments. Arch Hydrobiol 120:51-65

Gliwicz ZM, Lampert W (1990) Food thresholds in Daphnia species in the absence and presence of blue-green filaments. Ecology 71:691-702

Gliwicz ZM, Siedlar E (1980) Food size limitation and algae interfering with food collection in Daphnia. Arch Hydrobiol 88:155-177

Guillard RRL, Lorenzen CJ (1972) Yellow-green algae with chlorophyllide C. J Phycol 8:10-14

Haney JF (1987) Field studies on zooplankton-cyanobacteria interactions. N Z J Mar Freshw Res 21:467-475. doi:10. 1080/00288330.1987.9516242

Haney JF, Sasner JJ, Ikawa M (1995) Effects of products released by Aphanizomenon flos-aquae and purified saxitoxin on the movements of Daphnia carinata feeding appendages. Limnol Oceanogr 40:263-272. doi:10.4319/ 10.1995.40.2.0263

Hartmann JH, Kunkel DD (1991) Mechanisms of food selection in Daphnia. Hydrobiologia 225:129-154. doi:10.1007/ BF00028392

Hawkins P, Lampert W (1989) The effect of Daphnia body size on filtering rate inhibition in the presence of a filamentous 
cyanobacterium. Limnol Oceanogr 34:1084-1089. doi:10. 4319/1o.1989.34.6.1084

Hoiczyk E, Hansel A (2000) Cyanobacterial cell walls: news from an unusual prokaryotic envelope. J Bacteriol 182:1191-1199. doi:10.1128/JB.182.5.1191-1199.2000

Koehl MAR (1995) Fluid flow through hair-bearing appendages: feeding, smelling, and swimming at low and intermediate Reynolds number. Symp Soc Exp Biol 49:157-182

Komárek J (2013) Cyanoprokaryota: 3. Teil/3rd part: heterocytous genera. In: Büdel B, Gärtner G, Krienitz L, Schagerl L (eds) Süßwasserflora von Mitteleuropa, Bd. 19/3. Springer, Berlin

Koza V, Kořinek V (1985) Adaptability of the filtration screen in Daphnia: another answer to the selective pressure of the environment. Arch Hydrobiol Beih Ergebn Limnol 21: 193-198

Kurmayer R (2001) Competitive ability of Daphnia under dominance of non-toxic filamentous cyanobacteria. Hydrobiologia 442:279-289. doi:10.1023/A:101759 8518952

Lampert W (1987) Laboratory studies on zooplanktoncyanobacteria interactions. N Z J Mar Freshw Res 21:483-490. doi:10.1080/00288330.1987.9516244

Lampert W (1994) Phenotypic plasticity of the filter screens in Daphnia: adaptation to a low-food environment. Limnol Oceanogr 39:997-1006. doi:10.4319/lo.1994.39.5.0997

Lampert W, Brendelberger H (1996) Strategies of phenotypic low-food adaptation in Daphnia: filter screens, mesh sizes, and appendage beat rates. Limnol Oceanogr 41:216-223. doi:10.4319/lo.1996.41.2.0216

Lemaire V, Brusciotti S, van Gremberghe I, Vyverman W, Vanoverbeke J, De Meester L (2012) Genotype $\times$ genotype interactions between the toxic cyanobacterium $M i$ crocystis and its grazer, the waterflea Daphnia. Evol Appl 5:168-182. doi:10.1111/j.1752-4571.2011.00225.x

Macháček J (1998) What can we learn from Daphnia filtering screens? J Plankton Res 20:1645-1650. doi:10.1093/ plankt/20.8.1645

Pop M (1991) Mechanisms of the filtering area adaptation in Daphnia. Hydrobiologia 225:169-176. doi:10.1007/ BF00028394

R Core Team (2013) R: a language and environment for statistical computing. R. Foundation for statistical computing, Vienna. ISBN 3-900051-07-0. http://www.R-project.org/

Reinikainen M, Hietala J, Walls M (1999) Reproductive allocation in Daphnia exposed to toxic cyanobacteria. J Plankton Res 21:1553-1564

Repka S (1997) Effects of food type on the life history of Daphnia clones from lakes differing in trophic state. I. Daphnia galeata feeding on Scenedesmus and Oscillatoria. Freshw Biol 38:675-683
Repka S, Veen A, Vijverberg J (1999a) Morphological adaptations in filtering screens of Daphnia galeata to food quantity and food quality. J Plankton Res 21:971-989. doi:10.1093/plankt/21.5.971

Repka S, Veselá S, Weber A, Schwenk K (1999b) Plasticity in filtering screens of Daphnia cucullata $\times$ galeata hybrids and parental species at two food concentrations. Oecologia 120:485-491

Sahuquillo M, Melão MGG, Miracle MR (2007) Low filtering rates of Daphnia magna in a hypertrophic lake: laboratory and in situ experiments using synthetic microspheres. Hydrobiologia 594:141-152. doi:10.1007/s10750-0079079-0

Shafik HM, Vörös L, Sprőber P, Présing M, Kovács AW (2003) Some special morphological features of Cylindrospermopsis raciborskii in batch and continuous cultures. Hydrobiologia 506:163-167. doi:10.1023/B:HYDR. 0000008588.89048.70

Soares MCS, Lürling M, Panosso R, Huszar V (2009) Effects of the cyanobacterium Cylindrospermopsis raciborskii on feeding and life-history characteristics of the grazer Daphnia magna. Ecotoxicol Environ Saf 72:1183-1189. doi:10.1016/j.ecoenv.2008.09.004

Stearns SC (1992) The evolution of life histories. Oxford University Press, Oxford

Stewart I, Schluter PJ, Shaw GR (2006) Cyanobacterial lipopolysaccharides and human health-a review. Environ Health 5:7. doi:10.1186/1476-069X-5-7

Stuchlik E (1991) Feeding behaviour and morphology of filtering combs of Daphnia gaelata. Hydrobiologia 225:155-167. doi:10.1007/BF00028393

Wejnerowski L, Cerbin S, Dziuba MK (2015) Thicker filaments of Aphanizomenon gracile are more harmful to Daphnia than thinner Cylindrospermopsis raciborskii. Zool Stud 54:2. doi:10.1186/s40555-014-0084-5

Wejnerowski L, Cerbin S, Wojciechowicz MK, Dziuba M (2016) Differences in cell wall of thin and thick filaments of cyanobacterium Aphanizomenon gracile SAG 31.79 and their implications for different resistance to Daphnia grazing. J Limnol 75:634-643. doi:10.4081/jlimnol.2016. 1383

Zanchett G, Oliveira-Filho EC (2013) Cyanobacteria and cyanotoxins: from impacts on aquatic ecosystems and human health to anticarcinogenic effects. Toxins 5:1896-1917. doi:10.3390/toxins5101896

Zánkai PN, Ponyi JE (1986) Composition, density and feeding of crustacean zooplankton community in a shallow, temperate lake (Lake Balaton, Hungary). Hydrobiologia 135:131-147. doi:10.1007/BF00006465 